\title{
Modern design optimisation exploiting field simulation
}

\author{
Jan K. Sykulski \\ School of Electronics and Computer Science, University of Southampton \\ Southampton, SO17 1BJ, UK, e-mail: jks@soton.ac.uk
}

\begin{abstract}
The presentation will review some of the new developments in optimisation techniques and their relevance to the design of electrical machines and drive systems. Cost effective algorithms will be explored for computationally expensive modelling processes such as encountered when field simulation techniques are employed in CAD aided design. Surrogate modelling, kriging-assisted methods, pareto-optimality and design sensitivity will be emphasised.
\end{abstract}

Keywords-Design, modelling, simulation, optimisation.

\section{INTRODUCTION}

This review builds on previous publications by the author, in particular the overview presented at the last EPE-PEMC conference in Portoroz [1]. It was argued then that significant advances had taken place in the field of Computational Electromagnetics and demonstrated how numerical field simulation could aid the design of electrical machines and drive systems. Harnessing primarily the versatile finite element approach, the commercially available software - including general purpose packages - already offers a mature tool for performance prediction, optimisation and general design. Two particular challenges were also identified as a potential road map to successful design: modelling of the multi-physics problems and applying multi-objective optimisation. This review focuses on the latter and will relate to another review published last year addressing the emergence of new optimisation techniques for tackling computationally expensive modelling processes [2]. Both publications together contain a vast selection of relevant references and web addresses which the reader is encouraged to consult for details.

\section{HIERARCHICAL DESIGN}

There are many definitions of design, for our purposes the most appropriate is probably the one which describes design as a process of searching for a device or structure which satisfies a set of requirements. This is certainly an inverse problem where the requirements may be expressed in terms of the physical sizes, the inputs and/or the outputs, as well as possibly some special characteristics or properties.

A traditional ('trial and error') design typically consists of the following steps: (1) guess a solution, (2) build the device and measure its performance, (3) modify the device to more nearly match the requirements, (4) carry on improving until specification is met within acceptable tolerance. The modifications are usually performed on the basis of simple models, design expertise and "know-how".
A design engineer is expected to have an appreciation of how a change in a particular parameter will affect the device performance. In other words, he/she has a mental picture of how small changes in any parameter will affect each aspect of the desired performance. This is in fact a concept of sensitivity, which incidentally has a much wider application than just guiding a search, as it can be used to determine the effects of manufacturing errors (robustness of a design), it provides basis for finding performance parameters (e.g. force calculation) and it can be formulated as an optimisation method, an approach which will be pursued later in this talk.

Alternately, if no experience or models exist, random variations can be tried, the performance measured and appropriate models developed. However, the notion of 'virtual prototyping' becomes relevant here as it may be cheaper and more efficient to explore design space using computational (software) models rather than real (hardware) counterparts. Different levels of complexity of these computational models may be appropriate at different design stages, ranging from primitive equivalent circuits, through simple magnetic circuit representations, full numerical 2D and 3D formulations, to integrated field-circuit multi-physics system models. This leads us nicely into the concept of hierarchical design.

It is increasingly argued that the most efficient approach to design is to combine all available tools, methods and approaches - from very simple to advanced and use them in a logical way, the simplest of which is the top-down approach. Thus we start with a very large design space and use approximate but very fast solutions (e.g. equivalent circuits, semi-empirical, design sheets, etc) taking full advantage of extensive knowledge base available and conducting extensive optimisation. The design space is then progressively reduced so that more accurate, but computationally more expensive, models can be used, such as 2D finite element, static or steady-state. Some constrained optimisation may accompany, perhaps coupling to circuit models as well. Finally, for most devices, full 3D finite-element, often transient, simulation needs to be conducted, effectively providing a fine tuning of the design. Under this hierarchical structure all models of varied complexity have their important role to play, although the most important stage in the process appears to be the middle tier, where major decisions are taken and where the geometric and topological structure of the device is being shaped up. Although the models used here may not be the most accurate, they certainly offer better accuracy than the simplistic treatment of the top tier, although there may be a certain overlap between the first and the second stage in terms of models used. 
The essential part of the whole design process is optimisation and its most distinctive aspect is that it is computationally expensive as each calculation of the objective function typically requires a full finite element field solution, and often several such solutions (to find an average torque for example), or a solution to a coupled problem. Thus methods of optimisation relevant to this type of design must be efficient in requiring as few function calls as possible.

\section{SINGLE- AND MULTI-OBJECTIVE OPTIMISATION}

Central to this discussion is the so called 'no free lunch' (NFL) theorem which prohibits the existence of an algorithm which would outperform all other optimisation algorithms, when averaged over all possible problems. In other words, when averaged over an infinite number of all types of problems, every algorithm performs the same. However, design engineers are only interested in a subset of problems, thus - consistent with the NFL theorem - it is possible to identify a set of algorithms which outperform others over a particular domain of interest.

Single-objective optimization problems (SOOP) are well researched and various techniques are in abundance. Performance criteria used for comparison of algorithms include: best function value found, CPU time, number of function evaluations, accuracy, success rate and stopping criteria. In multi-objective optimization problems (MOOP) the aim is to simultaneously minimize a number of different objectives. This may be achieved using scalarizing methods, which combine the multiple objectives of the MOOP using some function, and then use one of the methods for single-objective optimization. Non-scalarizing methods, on the other hand, consider each objective function individually.

It is helpful to introduce the notion of pareto-optimality. Rather then a single optimal solution, we arrive at a set of possible designs forming a pareto-optimal front. For a multi-objective problem it is very unlikely that all objectives can achieve optimum simultaneously (the so called 'utopia point'), thus the final design is necessarily a compromise, but rather then making a priori decisions by combining objectives through for example weighting functions, we leave the final choice until after a posteriori assessment of all possible solutions.

Recent years have witnessed significant research effort in developing new techniques for optimisation of computationally intensive problems. These are mainly relying on surrogate modelling with various basis functions selected to model the reality. The surrogate model which stands out, due to its solid statistical foundations, is kriging, which is essentially as a Gaussian process characterized by its mean and its covariance function. In kriging-assisted SOOPs, the method of selecting search points may be two-stage, where first the surrogate model is fitted to the observed points and then a utility function is constructed to determine the next search point, or one-stage, where a design vector is determined which would yield the most credible response surface. Almost all existing algorithms are two-stage; however one-stage algorithms have been successfully constructed using both kriging and other surrogate models.

A detailed review of these new methods may be found in [2], and attention is drawn to the following recent SOOP techniques: Efficient Global Optimisation (EGO),
Generalized Expected Improvement (GEI), Weighted Expected Improvement (WEI), Credibility of a Hypothesis $(\mathrm{CH})$ and Minimizer Entropy (ME) criterion.

The scalarizing methods for converting MOOP into SOOP include: $\varepsilon$-constraint $(\varepsilon-C)$, Weighting method (W), Weighted Metrics (WM) (including the Tchebycheff metric) method, Achievement scalarizing Function approach (AF), Lexicographic Ordering approach (LO) and Value Function method (VF). The main purpose of a scalarizing function is to combine the multiple objectives of a MOOP in such a way that the contours of the resulting function are able to capture every point on the Pareto-optimal front.

It is interesting to note that there are a large number of selection criteria for SOOP and a large number of methods for transforming a MOOP to a SOOP, thus creating a huge number of scalarizing MOOP Algorithms (made possible with kriging), only very few of which have already been investigated in terms of their efficiency as practical tools.

Finally, all the techniques mentioned address the crucial aspect of any optimisation algorithm, that is striking a careful balance between exploitation and exploration.

\section{SENSITIVITY STUDIES}

Special attention needs to be paid to a class of methods based on sensitivity analysis, which offer the advantage of having computation times independent of the number of design variables, thus making them particularly useful in topology optimisation, where the parameterization enables all feasible shapes of electromagnetic devices to be explored. These algorithms are still at early stages of development and not versatile enough to be considered as competitive against surrogate modelling, but their performance is very impressive.

The sensitivity formula needs to be derived for both the primary system and the adjoint system. By exploring the analogy between the two formulations the geometric and material properties of the adjoint system are found to be the same as those of the primary system and sources may be recognised as electric current or permanent magnet. Thus the adjoint system - despite having been introduced as a mathematical derivation - is physically well based. An added benefit is that if the objective function is energy related the system becomes self-adjoint.

\section{CONCLUDING REMARKS}

The talk will focus on new developments in methods of optimisation relevant to the design of electrical machines and drive systems. Many efficient new approaches have recently been proposed, in particular for computationally intensive problems, and optimisation continues to be a very active area of research. Most existing design systems are based on algorithms developed in the $80 \mathrm{~s}$ and $90 \mathrm{~s}$, while the new techniques offer exciting new opportunities.

\section{REFERENCES}

[1] J. K. Sykulski, "Field Simulation as an Aid to Machine Design: the State of the Art," Proceedings of the 12th International Power Electronics and Motion Control Conference EPE-PEMC 2006, Portoroz, Slovenia, IEEE Catalog Number: 06EX1282C, Library of Congress: 2005938592, pp. 1937-1942, September 2006.

[2] J. K. Sykulski, "New trends in optimization in electromagnetics," Przeglad Elektrotechniczny, ISSN 0033-2097, vol. 83, pp. 13-18, June 2007. 